\title{
BMJ A meta-analysis of cognitive-based Open behaviour change techniques as interventions to improve medication adherence
}

\author{
Claire Easthall, ${ }^{1}$ Fujian Song, ${ }^{2}$ Debi Bhattacharya ${ }^{1}$
}

To cite: Easthall C, Song F, Bhattacharya D. A metaanalysis of cognitive-based behaviour change techniques as interventions to improve medication adherence. BMJ Open 2013;3:e002749. doi:10.1136/bmjopen-2013002749

- Prepublication history and additional material for this paper is available online. To view these files please visit the journal online (http://dx.doi.org/10.1136/ bmjopen-2013-002749)

Received 18 February 2013 Revised 1 July 2013

Accepted 3 July 2013

\begin{abstract}
${ }^{1}$ School of Pharmacy, University of East Anglia, Norwich Research Park, Norwich, UK

${ }^{2}$ Norwich Medical School, University of East Anglia, Norwich Research Park, Norwich, UK
\end{abstract}

Correspondence to Claire Easthall;

c.easthall@uea.ac.uk

\section{ABSTRACT}

Objective: To describe and evaluate the use of cognitive-based behaviour change techniques as interventions to improve medication adherence.

Design: Systematic review and meta-analysis of interventions to improve medication adherence.

Data sources: Search of the MEDLINE, EMBASE, PsycINFO, CINAHL and The Cochrane Library databases from the earliest year to April 2013 without language restriction. References of included studies were also screened to identify further relevant articles.

Review methods: We used predefined criteria to select randomised controlled trials describing a medication adherence intervention that used Motivational Interviewing (MI) or other cognitive-based techniques. Data were extracted and risk of bias was assessed by two independent reviewers. We conducted the meta-analysis using a random effects model and Hedges' $g$ as the measure of effect size.

Results: We included 26 studies (5216 participants) in the meta-analysis. Interventions most commonly used MI, but many used techniques such as aiming to increase the patient's confidence and sense of selfefficacy, encouraging support-seeking behaviours and challenging negative thoughts, which were not specifically categorised. Interventions were most commonly delivered from community-based settings by routine healthcare providers such as general practitioners and nurses. An effect size $(95 \% \mathrm{Cl})$ of $0.34(0.23$ to 0.46$)$ was calculated and was statistically significant $(p<0.001)$. Heterogeneity was high with an $\mathrm{I}^{2}$ value of $68 \%$. Adjustment for publication bias generated a more conservative estimate of summary effect size of 0.21 ( 0.08 to 0.33 ). The majority of subgroup analyses produced statistically nonsignificant results.

Conclusions: Cognitive-based behaviour change techniques are effective interventions eliciting improvements in medication adherence that are likely to be greater than the behavioural and educational interventions largely used in current practice. Subgroup analyses suggest that these interventions are amenable to use across different populations and in differing manners without loss of efficacy. These factors may facilitate incorporation of these techniques into routine care.

\section{ARTICLE SUMMARY}

Article focus

- Medication non-adherence is widespread and represents a notable barrier in achieving optimal effects from therapeutic intervention.

- Despite the magnitude and consequences of non-adherence, a gold standard intervention to improve it remains elusive.

- Cognitive-based behaviour change techniques (CBCTs) may represent a useful tool in improving medication adherence, but their use in this domain has not been established using meta-analytic techniques.

Key messages

- CBCTs are effective interventions for improving medication adherence and capable of eliciting improvements in adherence beyond those achieved with educational and behavioural interventions which form the mainstay of current practice.

- According to the results of subgroup analyses, CBCTs can be effectively delivered by routine healthcare providers, and the effectiveness of interventions is not associated with intervention exposure.

- Healthcare providers may wish to consider incorporation of these techniques into their medication adherence consultations.

Strengths and limitations of this study

- The studies pooled in this meta-analysis are restricted to randomised controlled trials, which strengthens their robustness.

- Techniques to account for publication bias have been utilised to provide a conservative effect size estimate offering robustness to our estimate.

- Notable heterogeneity was reported when studies were combined, which may be a limitation.

\section{INTRODUCTION}

Estimates suggest that $30-50 \%$ of patients prescribed medications for chronic illnesses do not adhere to their prescribed medication regimen. ${ }^{1}$ This non-adherence has been 
demonstrated to diminish treatment effect which can result in prolonged illness, additional investigations and prescriptions that may otherwise have been unnecessary. ${ }^{2}$ A link between poor adherence and an increased risk of mortality is also well established. ${ }^{3}$ Consequently, the WHO has described non-adherence as 'a worldwide problem of striking magnitude' and a priority for healthcare researchers and policymakers. ${ }^{1}$

Despite the magnitude and potential gravity of suboptimal medication adherence, a gold standard intervention remains elusive; a recent Cochrane review highlighted the paucity of effective interventions in current practice. ${ }^{4}$ Evidence suggests that complex, multifaceted interventions tailored to meet individual needs are most likely to be efficacious, ${ }^{4}{ }^{5}$ which is intuitive given the complex, multistage process that is taking medication.

Non-adherent behaviour is traditionally categorised into unintentional and intentional. Unintentional nonadherence includes behaviours arising from forgetfulness, misunderstanding and confusion. Intentional nonadherence describes patient choice that deviates from the prescribed medication regimen. Unintentional and intentional non-adherence are not mutually exclusive; thus, an amalgam of these behaviours often exists in any one patient. An understanding of patient behaviour and its underpinning psychology plus the wealth of factors, both internal and external that may influence medication taking, is crucial to understand how to change patient behaviour and thus improve medication adherence. ${ }^{6}$

Historically, adherence interventions have encompassed behaviour change techniques such as simplifying dosage regimens and providing adherence aids or education to address the practical issues of adherence in terms of knowing how and being able to take the medication as prescribed. Pooled data for such studies have demonstrated marginal effects, ${ }^{4}$ yet such interventions continue to form the cornerstone of routine healthcare provision. $^{2}$ These interventions may have particularly poor efficacy in cases of intentional non-adherence as the provision of persuasive advice may evoke further resistance to change. ${ }^{78}$ Through an understanding of the challenges faced in changing behaviours and the motivation necessary to achieve change, novel, cognitivebased behaviour change techniques (CBCTs) have emerged. These interventions aim to change a patient's behaviour by altering their thoughts, feelings, confidence or motivation to adhere. CBCT interventions can vary widely in content such as incorporating techniques to enhance patient sense of self-efficacy, problem solve and increase motivation to adhere.

Motivational Interviewing (MI) is one of the most widely recognised CBCTs and is designed to facilitate behaviour change by resolving patient ambivalence about change. ${ }^{9}$ It therefore primarily targets intentional non-adherence but also enables patients to reflect on any unintentional barriers to adherence and seek out solutions. Systematic reviews and meta-analyses have reported MI efficacy in facilitating health-related behaviour change such as smoking cessation and alcohol withdrawal $^{10-16}$ but have not explored its effects on medication adherence. Adaptations of MI such as Behaviour Change Counselling ${ }^{17}$ additionally allow the facilitator to educate and advise, and thus application to both intentional and unintentional non-adherence may be effective.

Best practice guidelines state that evidence of intervention efficacy should ideally be pooled from the literature in a systematic review or meta-analysis wherever possible to offer a robust and cohesive evidence base. ${ }^{18}$ This study provides a systematic review and meta-analysis of MI and other cognitive-based techniques as interventions to improve medication adherence.

\section{METHODS}

We used standard systematic review methods ${ }^{18} 19$ and registered the study protocol (PROSPERO register reference CRD42011001721). Randomised controlled trials (RCTs) reporting an adherence intervention using MI and/or other cognitive-based techniques with medication adherence as an outcome measure were eligible for inclusion. All definitions of adherence, such as the percentage of doses taken over a given time period and percentage of patients achieving a specified adherence level, were considered. All adherence measures were also considered, including self-report and electronic monitoring. Where multiple measures were reported, the percentage of patients achieving a specified adherence level was selected as this was common to more studies.

Any intervention using some form of psychological technique to change a patient's adherence behaviour, as well as their thoughts, feelings, confidence or motivation towards adherence, was defined as a cognitive-based technique. Studies examining adherence to medications for the treatment of addiction and/or mental health conditions were excluded as these interventions tend to be specific to these domains.

\section{Search strategies}

We developed a search strategy to avoid restriction to predetermined terms such as 'motivational interviewing' as many of the techniques of interest are not classified using specific or consistent terms. MeSH terms were also used to enhance retrieval of relevant studies. Truncations $(*)$, wild cards $(\$)$, hyphens and other relevant Boolean operators were used where permitted. Scoping searches were conducted prior to finalising the search strategy to ensure suitability of terms in generating a good coverage of relevant material.

We applied the search strategy (as shown in online supplementary appendix 1) to the MEDLINE, EMBASE, PsychINFO, CINAHL and Cochrane databases in April 2013 without date or language restrictions. The 
reference lists of all screened full-text articles were also used to identify further relevant articles.

\section{Study selection and data extraction}

Two researchers (CE and EP) independently screened titles and abstracts against the inclusion and exclusion criteria using a piloted abstract screening tool. Inter-reviewer agreement using Cohen's $\kappa$ was assessed for the abstract and full-text screening stages. The level of agreement was characterised using a qualitative scale. $^{20}$ Discrepancies were resolved by discussion between the two reviewers and, if necessary, referral to a third independent reviewer (DB) until consensus was reached.

Data extraction was also undertaken by $\mathrm{CE}$ and EP, independently using piloted forms. Data extracted included study details (such as year and journal of publication, country and study design); study characteristics (including setting, population, delivery methods and personnel); intervention details (including intervention type, duration and principal components) and outcome details (including adherence assessment measure, data and definition). A list of intervention components was independently extracted from the articles verbatim by two reviewers. Grouping of similar components was undertaken by one reviewer and verified by a second reviewer.

Accuracy of data collected was verified by comparison of the forms completed by the two independent reviewers. In cases of discrepancy, consensus was agreed through discussion and, where necessary, referral to a third independent reviewer (DB). For studies with missing data or ambiguities, the corresponding author was contacted for clarification.

\section{Quality assessment}

A quality assessment of all included studies was made using the Cochrane risk of bias tool. ${ }^{18}$ The risk of bias was assessed in five domains deemed relevant to the included studies: random sequence generation, allocation concealment, blinding of outcome assessment, incomplete outcome data and selective reporting. Performance bias (blinding of participants and personnel) was not included as the nature of the interventions meant that blinding of participants and personnel was impossible in almost all studies. None of the included studies were found to contain additional sources of potential bias not represented by the five included domains. The risk of bias for each study, in each of the five domains, was classified as low, uncertain or high, as recommended in the guidelines. ${ }^{18}$ The quality assessment process was undertaken independently by two reviewers, with consensus on the final risk classifications reached through discussion.

\section{Data analysis}

The meta-analysis was conducted using STATA (V.12.1). Given the broad inclusion criteria, we anticipated including studies from different populations with different diseases and which used different CBCTs. We therefore explored heterogeneity via calculation of the $\mathrm{I}^{2}$ statistic, which describes the percentage of total variation across studies that is due to heterogeneity rather than chance. ${ }^{21}{ }^{22}$ A random effects model (DerSimonian-Laird method) was employed to calculate a pooled effect size (Hedges' g) and 95\% CI for the included studies. ${ }^{23}$ Calculation of the effect size as Hedges' $g$ (standardised difference in means) enabled adherence outcome measures of differing definition and measure to be combined, transforming this data into a common metric. When SD was missing, we estimated SE of mean difference based on reported $p$ values, means and the number of patients. ORs were converted to standardised mean differences by using the formula $\mathrm{SMD}=\operatorname{lnOR} \times \sqrt{ } 3 / \pi^{23}$

Funnel plots were produced where appropriate to explore potential publication biases. STATA (V.12.1) was used to conduct Egger's test ${ }^{24}$ to test funnel plot asymmetry. We used the trim-and-fill method ${ }^{25} 26$ to estimate a summary effect size after adjusting for asymmetric funnel plots.

Variables of interest in influencing the effect size and informing intervention design were determined a priori and the following subgroup analyses undertaken using a random effects meta-regression: intervention components, setting, delivery personnel, delivery method and intervention exposure, disease area and risk of bias. The type of outcome measure used to assess adherence (objective compared with subjective) was added as a post hoc subgroup analysis to further explore heterogeneity. Objective outcome measures included electronic monitoring and pill counts, while subjective measures included all forms of self-report. Differences between subgroups were tested using the STATA 'metareg' command for random-effects univariate meta-regression analysis.

\section{RESULTS}

\section{Study selection, characteristics and quality}

Figure 1 shows the number of papers excluded at each stage of the review. Of the 442 abstracts screened, 84 studies passed the abstract screening stage with moderate agreement between the two reviewers $(\kappa=0.57)$. Conflict in classifying an intervention as a CBCT accounted for $31 \%$ of discrepancies and was heavily influenced by a paucity of information in the abstracts. At the full-text screening stage, agreement between the two independent reviewers was much higher, with a $\mathrm{k}$ value of 0.91 , indicating almost perfect agreement. After examining 84 full-text articles, we included $26(31 \%)$ in the meta-analysis.

The main characteristics of the 26 included studies are summarised in table 1 . The studies provided a total sample size of 5216 participants and were primarily undertaken in the USA, followed by the $\mathrm{UK},{ }^{27-29}$ Australia $^{30}{ }^{31}$ and the Netherlands. ${ }^{32}{ }^{33}$ Dates of publication ranged from 1990 to 2012 with only two studies 


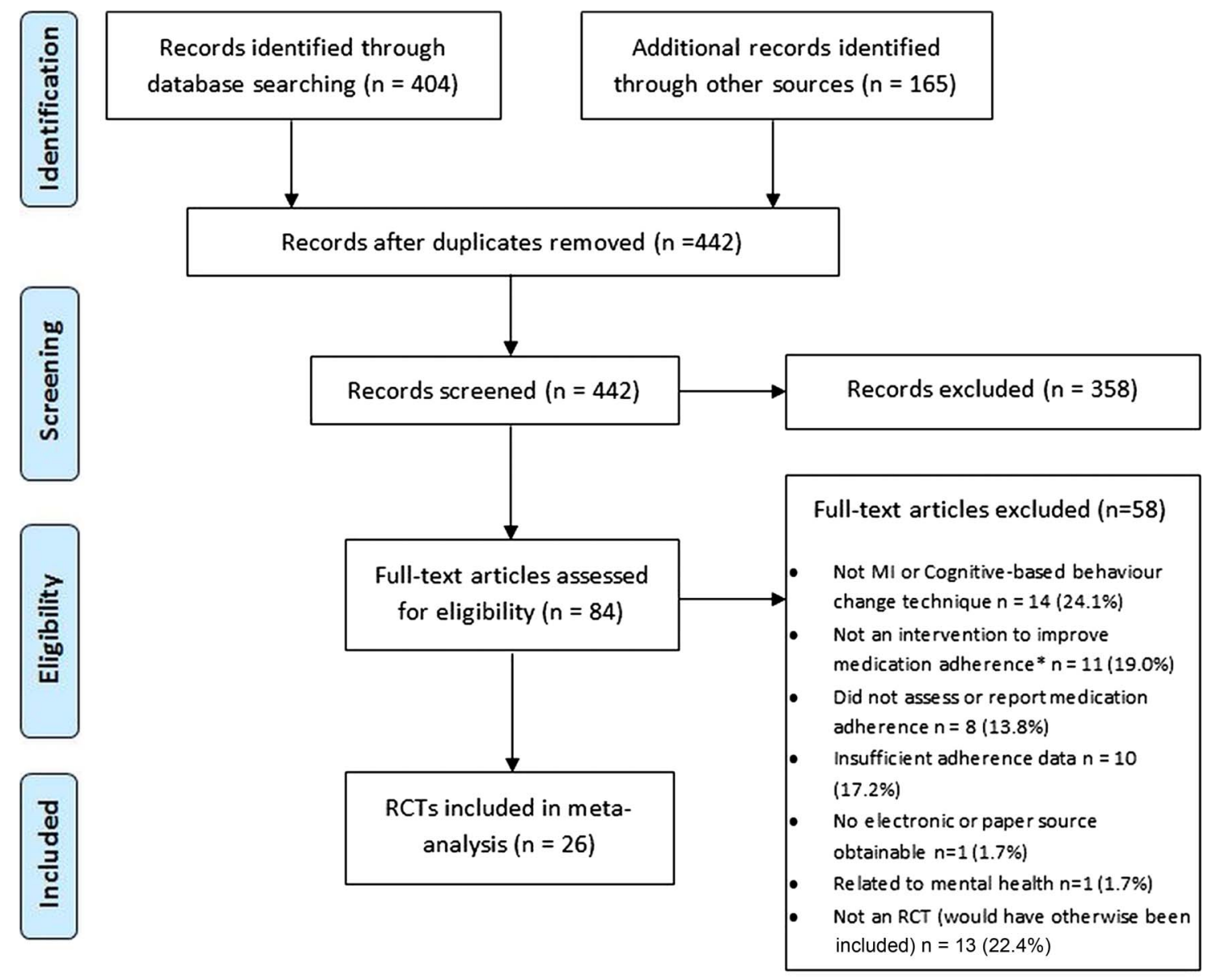

* Includes full texts that were not journal articles e.g. thesis chapters and reviews

Figure 1 Flow diagram for selection of studies.

(7.7\%) predating 2000. ${ }^{28} 34$ Ten $(38.5 \%)$ were published within the last 5 years (2008-2013). The most common condition for which medications were prescribed was HIV, accounting for $14(53.8 \%)$ studies. Other studies concerned treatments for a range of conditions including asthma, ${ }^{32} 3435$ diabetes $^{27} 31$ and hypertension. ${ }^{30} 36$

Just over half of the included studies $(53.8 \%)$ described an intervention with a clearly defined CBCT; MI was most commonly used and this was the case for $11(42.3 \%)$ studies. ${ }^{30} 31{ }^{36-44}$ A further three (11.5\%) studies used Implementation Intention Interventions (III, also known as if-then planning) as a clearly defined CBCT. For $12(46.2 \%)$ studies, a clearly defined CBCT, such as MI, could not be identified; ${ }^{32-35}{ }^{45-52}$ these studies are identified in table 1 as 'multiple components; non-specific techniques'. Instead, this group comprised multiple components such as 'providing education' or 'increasing patient knowledge', which was reported in nine $(75 \%)$ studies in this group. Other components included 'increasing self-efficacy' and 'developing or improving problem solving skills', each reported in six (50) studies and 'identifying and resolving adherence barriers' and 'increasing social support', each of which was also reported in six studies $(50 \%)$. All studies within this group included one or more components that aimed to alter the patient's thoughts, feelings, motivation or confidence towards adherence and that could therefore be classified as a CBCT. Detailed information regarding the identified intervention components extracted from each study are provided as an online supplementary table. The majority of interventions had multiple components. Many studies combined CBCT with more traditionally used educational (eg, increasing patient knowledge) and behavioural (eg, regimen simplification and provision of dosing aids) components.

Interventions were most commonly delivered in person, from community-based settings and by routine healthcare providers such as nurses, pharmacists and general medical practitioners. 'Non-routine' healthcare providers were considered to be those such as psychologists or psychotherapists, who would not ordinarily be involved in the patient's care.

The intervention period ranged from four $(15.4 \%)$ studies reporting singular sessions, to six $(23 \cdot 1 \%)$ studies reporting multiple sessions over 12 months. The median (IQ) number of sessions over which interventions were delivered was 5 (3-7.3). The majority of interventions were delivered over a period of 6 months or 


\begin{tabular}{|c|c|c|c|c|c|c|c|c|}
\hline Study & Study setting & $\begin{array}{l}\text { Disease } \\
\text { area }\end{array}$ & Intervention description* & $\begin{array}{l}\text { Identified } \\
\text { intervention } \\
\text { components } \\
\end{array}$ & $\begin{array}{l}\text { Components } \\
\text { received by } \\
\text { control group }\end{array}$ & $\begin{array}{l}\text { Sample } \\
\text { size }\end{array}$ & $\begin{array}{l}\text { Intervention } \\
\text { delivery style } \\
\text { (and } \\
\text { personnel) }\end{array}$ & $\begin{array}{l}\text { Intervention } \\
\text { length } \\
\text { (average) }\end{array}$ \\
\hline Bailey et $a{ }^{\beta 4}$ & $\begin{array}{l}\text { Hospital clinic, } \\
\text { USA }\end{array}$ & Asthma & $\begin{array}{l}\text { Comprehensive programme } \\
\text { integrating a skill-orientated } \\
\text { self-help workbook with one-to-one } \\
\text { counselling and } \\
\text { adherence-enhancing strategies }\end{array}$ & $\begin{array}{l}\text { Multiple components; } \\
\text { non-specific } \\
\text { techniques }\end{array}$ & $\begin{array}{l}\text { Standard care; } \\
\text { education via a } \\
\text { standardised } \\
\text { set of } \\
\text { pamphlets and } \\
\text { routine } \\
\text { physician } \\
\text { encouragement }\end{array}$ & 225 & $\begin{array}{l}\text { Telephone } \\
\text { calls and in } \\
\text { person } \\
\text { (specialist) }\end{array}$ & $\begin{array}{l}240 \mathrm{~min} \\
(4 \times 60 \mathrm{~min} \\
\text { sessions) over } \\
\text { unknown } \\
\text { period }\end{array}$ \\
\hline Berger et $a t^{40}$ & $\begin{array}{l}\text { Telephone calls to } \\
\text { patients at home, } \\
\text { USA }\end{array}$ & $\begin{array}{l}\text { Multiple } \\
\text { sclerosis }\end{array}$ & $\begin{array}{l}\text { Software-supported intervention } \\
\text { based on transtheoretical model of } \\
\text { change and MI }\end{array}$ & $\begin{array}{l}\text { Motivational } \\
\text { Interviewing (MI) }\end{array}$ & $\begin{array}{l}\text { Standard care } \\
\text { plus could } \\
\text { telephone help } \\
\text { line }\end{array}$ & 367 & $\begin{array}{l}\text { Telephone } \\
\text { calls } \\
\text { (researcher) }\end{array}$ & $\begin{array}{l}9 \text { sessions of } \\
\text { unknown } \\
\text { duration } \\
\text { delivered over } \\
3 \text { months }\end{array}$ \\
\hline Brown et $a^{29}$ & Hospital clinic, UK & Epilepsy & $\begin{array}{l}\text { Formation of III via completion of a } \\
\text { self-administered questionnaire }\end{array}$ & $\begin{array}{l}\text { Implementation } \\
\text { Intention } \\
\text { Interventions (III) }\end{array}$ & $\begin{array}{l}\text { Standard care } \\
\text { plus self-report } \\
\text { questionnaires }\end{array}$ & 69 & $\begin{array}{l}\text { Questionnaire } \\
\text { completion } \\
\text { (not in person) }\end{array}$ & $\begin{array}{l}\text { One-off } \\
\text { intervention of } \\
\text { unknown } \\
\text { duration }\end{array}$ \\
\hline Dilorio et $a{ }^{41}$ & $\begin{array}{l}\text { Community clinic, } \\
\text { USA }\end{array}$ & HIV & $\begin{array}{l}\text { One-to-one counselling sessions } \\
\text { based on MI }\end{array}$ & MI & $\begin{array}{l}\text { Standard care; } \\
\text { usual } \\
\text { adherence } \\
\text { education } \\
\text { provided in the } \\
\text { clinic }\end{array}$ & 17 & $\begin{array}{l}\text { In person } \\
\text { (routine } \mathrm{HCP} \text { ) }\end{array}$ & $\begin{array}{l}5 \times 35 \text { min } \\
\text { sessions } \\
\text { delivered over } \\
12 \text { months }\end{array}$ \\
\hline Dilorio et $a f^{42}$ & $\begin{array}{l}\text { Hospital clinic, } \\
\text { USA }\end{array}$ & HIV & $\begin{array}{l}\text { MI as individual counselling } \\
\text { sessions }\end{array}$ & $\mathrm{Ml}$ & $\begin{array}{l}\text { Standard care; } \\
\text { usual } \\
\text { (extensive) } \\
\text { education } \\
\text { provided at the } \\
\text { clinic }\end{array}$ & 213 & $\begin{array}{l}\text { Mostly in } \\
\text { person with } \\
\text { some } \\
\text { telephone } \\
\text { calls (routine } \\
\text { HCP) }\end{array}$ & $\begin{array}{l}5 \text { sessions of } \\
35 \text { min over } \\
12 \text { months }\end{array}$ \\
\hline Farmer et $a^{P^{7}}$ & $\begin{array}{l}\text { Community-based } \\
\text { clinic, UK }\end{array}$ & $\begin{array}{l}\text { Type } 2 \\
\text { diabetes }\end{array}$ & $\begin{array}{l}\text { Brief intervention to elicit beliefs, } \\
\text { resolve barriers and form 'if-then' } \\
\text { plans }\end{array}$ & If-then planning (III) & $\begin{array}{l}\text { Standard care } \\
\text { plus additional } \\
\text { clinic visits for } \\
\text { blood tests }\end{array}$ & 211 & $\begin{array}{l}\text { In person } \\
\text { (clinic nurse) }\end{array}$ & $\begin{array}{l}\text { One-off } \\
\text { session } \\
\text { lasting } 30 \mathrm{~min} .\end{array}$ \\
\hline George et $a l^{30}$ & $\begin{array}{l}\text { Community } \\
\text { pharmacies, } \\
\text { Australia and } \\
\text { Tasmania }\end{array}$ & Hypertension & $\begin{array}{l}\text { Community pharmacy intervention } \\
\text { of one-to-one sessions, monitoring } \\
\text { and medication review }\end{array}$ & $\mathrm{Ml}$ & Standard care & 343 & $\begin{array}{l}\text { In person } \\
\text { (routine HCP) }\end{array}$ & $\begin{array}{l}3 \text { sessions of } \\
\text { unknown } \\
\text { duration over } \\
6 \text { months }\end{array}$ \\
\hline
\end{tabular}

Continued 


\begin{tabular}{|c|c|c|c|c|c|c|c|c|}
\hline Study & Study setting & $\begin{array}{l}\text { Disease } \\
\text { area }\end{array}$ & Intervention description* & $\begin{array}{l}\text { Identified } \\
\text { intervention } \\
\text { components }\end{array}$ & $\begin{array}{l}\text { Components } \\
\text { received by } \\
\text { control group }\end{array}$ & $\begin{array}{l}\text { Sample } \\
\text { size }\end{array}$ & $\begin{array}{l}\text { Intervention } \\
\text { delivery style } \\
\text { (and } \\
\text { personnel) }\end{array}$ & $\begin{array}{l}\text { Intervention } \\
\text { length } \\
\text { (average) }\end{array}$ \\
\hline Golin et $a \beta^{\beta 9}$ & $\begin{array}{l}\text { Community clinic, } \\
\text { USA }\end{array}$ & HIV & $\begin{array}{l}\text { Multicomponent MI-based } \\
\text { intervention }\end{array}$ & MI & $\begin{array}{l}\text { General HIV } \\
\text { information } \\
\text { provided via } \\
\text { audio tape, two } \\
\text { one-to-one } \\
\text { sessions and } \\
\text { two mail shots }\end{array}$ & 117 & $\begin{array}{l}\text { In person } \\
\text { (specialist) }\end{array}$ & $\begin{array}{l}2 \text { sessions of } \\
\text { unknown } \\
\text { duration over } \\
2 \text { months }\end{array}$ \\
\hline Hovell et $a^{51}$ & $\begin{array}{l}\text { Hospital clinic, } \\
\text { USA }\end{array}$ & Tuberculosis & $\begin{array}{l}\text { Adherence coaching involving } \\
\text { interviewing, contingency } \\
\text { contracting and shaping } \\
\text { procedures }\end{array}$ & $\begin{array}{l}\text { Multiple components; } \\
\text { non-specific } \\
\text { techniques }\end{array}$ & $\begin{array}{l}\text { Standard care; } \\
\text { routine advice } \\
\text { at appointments }\end{array}$ & 188 & $\begin{array}{l}\text { Telephone } \\
\text { calls and in } \\
\text { person } \\
\text { (researcher) }\end{array}$ & $\begin{array}{l}12 \text { sessions of } \\
15-30 \text { min } \\
\text { over } 6 \text { months }\end{array}$ \\
\hline $\begin{array}{l}\text { Konkle-Parker } \\
\text { et }\left.a\right|^{\beta 8}\end{array}$ & $\begin{array}{l}\text { Community-based } \\
\text { clinics and } \\
\text { patients' own } \\
\text { homes, USA }\end{array}$ & HIV & $\begin{array}{l}\text { Adherence intervention guided by } \\
\text { the } \\
\text { Information-Motivation-Behavioural } \\
\text { Skills (IMB) model }\end{array}$ & $\mathrm{Ml}$ & $\begin{array}{l}\text { Standard care; } \\
\text { usual clinic } \\
\text { appointments }\end{array}$ & 36 & $\begin{array}{l}\text { Telephone } \\
\text { calls and in } \\
\text { person (nurse } \\
\text { practitioner) }\end{array}$ & $\begin{array}{l}8 \text { sessions } \\
\text { over } \\
24 \text { weeks. } \\
\text { Average } \\
\text { overall } \\
\text { duration } 1 \mathrm{~h} \\
30 \mathrm{~min}\end{array}$ \\
\hline $\begin{array}{l}\text { Maneesriwongul } \\
\text { et } a \beta^{37}\end{array}$ & $\begin{array}{l}\text { Hospital } \\
\text { outpatients clinic } \\
\text { and telephone } \\
\text { calls to patients at } \\
\text { home, Thailand }\end{array}$ & HIV & MI with counselling & MI & $\begin{array}{l}\text { Standard care; } \\
\text { education and } \\
\text { provision of } \\
\text { leaflets at point } \\
\text { of prescribing }\end{array}$ & 60 & $\begin{array}{l}\text { Telephone } \\
\text { calls and in } \\
\text { person } \\
\text { (researcher) }\end{array}$ & $\begin{array}{l}3 \text { sessions } \\
\text { approximately } \\
30 \text { min over a } \\
4 \text { week period }\end{array}$ \\
\hline Murphy et $a^{\Gamma^{2}}$ & $\begin{array}{l}\text { Community-based } \\
\text { clinic, USA }\end{array}$ & HIV & $\begin{array}{l}\text { Multicomponent and } \\
\text { multidisciplinary intervention } \\
\text { including behavioural strategies } \\
\text { and cognitive behavioural therapy }\end{array}$ & $\begin{array}{l}\text { Multiple components; } \\
\text { non-specific } \\
\text { techniques }\end{array}$ & $\begin{array}{l}\text { Standard care; } \\
\text { regular } \\
\text { appointments } \\
\text { with enquiries } \\
\text { about } \\
\text { adherence and } \\
\text { an additional } \\
30 \text { min } \\
\text { appointment for } \\
\text { those with } \\
\text { problems where } \\
\text { medication } \\
\text { schedule is } \\
\text { written down for } \\
\text { them }\end{array}$ & 33 & $\begin{array}{l}\text { In person } \\
\text { (specialist) }\end{array}$ & $\begin{array}{l}5 \text { sessions of } \\
\text { unknown } \\
\text { duration over } \\
7 \text { weeks }\end{array}$ \\
\hline
\end{tabular}




\begin{tabular}{|c|c|c|c|c|c|c|c|c|}
\hline Study & Study setting & $\begin{array}{l}\text { Disease } \\
\text { area }\end{array}$ & Intervention description* & $\begin{array}{l}\text { Identified } \\
\text { intervention } \\
\text { components } \\
\end{array}$ & $\begin{array}{l}\text { Components } \\
\text { received by } \\
\text { control group }\end{array}$ & $\begin{array}{l}\text { Sample } \\
\text { size }\end{array}$ & $\begin{array}{l}\text { Intervention } \\
\text { delivery style } \\
\text { (and } \\
\text { personnel) }\end{array}$ & $\begin{array}{l}\text { Intervention } \\
\text { length } \\
\text { (average) }\end{array}$ \\
\hline $\begin{array}{l}\text { Ogedegbe } \\
\text { et } a{ }^{\beta 6}\end{array}$ & $\begin{array}{l}\text { Community clinic, } \\
\text { USA }\end{array}$ & Hypertension & Practice-based MI counseling & MI & $\begin{array}{l}\text { Standard care; } \\
\text { usual } \\
\text { appointments } \\
\text { plus additional } \\
\text { visits for MEMS } \\
\text { downloads }\end{array}$ & 160 & $\begin{array}{l}\text { In person } \\
\text { (researcher) }\end{array}$ & $\begin{array}{l}4 \text { sessions } \\
\text { lasting } 30- \\
40 \text { min } \\
\text { delivered over } \\
12 \text { months }\end{array}$ \\
\hline Pradier et $a^{50}$ & $\begin{array}{l}\text { Hospital clinic, } \\
\text { France }\end{array}$ & HIV & $\begin{array}{l}\text { Educational and counselling } \\
\text { intervention founded in the } \\
\text { principles of motivational } \\
\text { psychology and client-centred } \\
\text { therapy }\end{array}$ & $\begin{array}{l}\text { Multiple components; } \\
\text { non-specific } \\
\text { techniques }\end{array}$ & $\begin{array}{l}\text { Standard care; } \\
\text { routine } \\
\text { follow-up } \\
\text { appointments }\end{array}$ & 202 & $\begin{array}{l}\text { In person } \\
\text { (routine HCP) }\end{array}$ & $\begin{array}{l}3 \text { sessions of } \\
45-60 \text { min } \\
\text { over } 3 \text { months }\end{array}$ \\
\hline Put et $a{ }^{\beta 5}$ & $\begin{array}{l}\text { Hospital clinic, } \\
\text { Belgium }\end{array}$ & Asthma & $\begin{array}{l}\text { Behavioural change intervention } \\
\text { involving psycho-education with } \\
\text { behavioural and cognitive } \\
\text { techniques }\end{array}$ & $\begin{array}{l}\text { Multiple components; } \\
\text { non-specific } \\
\text { techniques }\end{array}$ & $\begin{array}{l}\text { Standard (no } \\
\text { details } \\
\text { provided) }\end{array}$ & 23 & $\begin{array}{l}\text { In person } \\
\text { (researcher) }\end{array}$ & $\begin{array}{l}360 \text { min } \\
(6 \times 60 \text { min } \\
\text { sessions }) \text { over } \\
3 \text { months }\end{array}$ \\
\hline Remien et $a /^{49}$ & $\begin{array}{l}\text { Community-based } \\
\text { clinic, USA }\end{array}$ & HIV & $\begin{array}{l}\text { Couples-based intervention } \\
\text { grounded in social action theory }\end{array}$ & $\begin{array}{l}\text { Multiple components; } \\
\text { non-specific } \\
\text { techniques }\end{array}$ & $\begin{array}{l}\text { Standard care; } \\
\text { education at } \\
\text { point of } \\
\text { prescribing and } \\
\text { follow-up to } \\
\text { check } \\
\text { adherence and } \\
\text { investigate/ } \\
\text { address } \\
\text { underlying } \\
\text { causes of any } \\
\text { non-adherence }\end{array}$ & 196 & $\begin{array}{l}\text { In person } \\
\text { (routine } \mathrm{HCP} \text { ) }\end{array}$ & $\begin{array}{l}4 \text { sessions of } \\
45-60 \text { min } \\
\text { over } 5 \text { weeks }\end{array}$ \\
\hline Safren et $a{ }^{44}$ & $\begin{array}{l}\text { Community clinic, } \\
\text { USA }\end{array}$ & HIV & $\begin{array}{l}\text { Single session minimal treatment } \\
\text { intervention using cognitive } \\
\text { behavioural, Ml and problem } \\
\text { solving techniques }\end{array}$ & MI & $\begin{array}{l}\text { Minimal contact } \\
\text { intervention; } \\
\text { daily diary used } \\
\text { to record no. of } \\
\text { pills prescribed } \\
\text { and taken each } \\
\text { day }\end{array}$ & 53 & $\begin{array}{l}\text { In person } \\
\text { (routine HCP) }\end{array}$ & $\begin{array}{l}\text { One-off } \\
\text { intervention of } \\
\text { unknown } \\
\text { duration }\end{array}$ \\
\hline
\end{tabular}


Simoni et a $\mathrm{f}^{48} \quad$ Community-based HIV clinic and

telephone calls to patients at home

USA

Smith et $a^{47} \quad$ Community-based HIV research office, USA
Peer-led medication-related social support intervention

Self-management intervention based on feedback of adherence performance and principles of social cognitive theory
Formation of III via completion of a Implementation

Solomon et $a f^{43} \quad$ Telephone calls to Osteoporosis Telephone-based counselling patient's own home, USA programme rooted in $\mathrm{MI}$

Hospital clinic, HIV Spain

HIV

Hospital clinic, Asthma Netherlands van Es et $a l^{\beta 2}$ on Self-efficacy theory

Intervention programme to stimulate a positive attitude, increase social support and enhance self-efficacy
Psycheducative intervention based

\section{Identified \\ intervention \\ components} Intention Intervention (III)

Multiple-components;
non-specific
techniques
non-specific techniques

MI

Intervention

$\begin{array}{lll}\text { Components } & & \begin{array}{l}\text { delivery style } \\ \text { received by }\end{array} \text { Samplervention } \\ \text { (and } & \text { length }\end{array}$

control group size

personnel)

(average)

Completion of 78

Questionnaire One-off

completion intervention of (not in person) unknown

questionnaire

but without

duration

formation of

implementation

intention

education

programme and

social and

health referrals

as necessary

Standard care; 17

usual

medication

counselling,

educational

leaflets,

scheduling

support

reminder lists

and discussion

of adherence

strategies

Standard care 2087 Telephone 8 sessions of

plus seven

information

mailings on

osteoarthritis

care

Multiple components; Standard care; 77 non-specific normal clinical

techniques

follow-up

Multiple components; Standard care; 67

non-specific routine

techniques check-ups
Group

18 sessions of

sessions and unknown

individual duration over

telephone 3 months

calls (peers)

In person

our sessions

of unknown

duration over

12 weeks 


\begin{tabular}{|c|c|c|c|c|c|c|c|c|}
\hline Study & Study setting & $\begin{array}{l}\text { Disease } \\
\text { area }\end{array}$ & Intervention description* & $\begin{array}{l}\text { Identified } \\
\text { intervention } \\
\text { components }\end{array}$ & $\begin{array}{l}\text { Components } \\
\text { received by } \\
\text { control group }\end{array}$ & $\begin{array}{l}\text { Sample } \\
\text { size }\end{array}$ & $\begin{array}{l}\text { Intervention } \\
\text { delivery style } \\
\text { (and } \\
\text { personnel) }\end{array}$ & $\begin{array}{l}\text { Intervention } \\
\text { length } \\
\text { (average) }\end{array}$ \\
\hline Wagner et al ${ }^{45}$ & $\begin{array}{l}\text { Community clinic, } \\
\text { USA }\end{array}$ & HIV & $\begin{array}{l}\text { Cognitive behavioural intervention } \\
\text { with motivational components, } \\
\text { based on the } \\
\text { information-motivation-behavioural } \\
\text { skills (IMB) model }\end{array}$ & $\begin{array}{l}\text { Multiple components; } \\
\text { non-specific } \\
\text { techniques }\end{array}$ & $\begin{array}{l}\text { Standard care } \\
\text { practices for } \\
\text { improving } \\
\text { adherence; } \\
\text { education, } \\
\text { tailoring } \\
\text { regimen, } \\
\text { offering a } \\
\text { pillbox, } \\
\text { adherence } \\
\text { checks and } \\
\text { enquiries about } \\
\text { side effects }\end{array}$ & 135 & $\begin{array}{l}\text { In person } \\
\text { (routine HCP) }\end{array}$ & $\begin{array}{l}5 \text { sessions of } \\
30-45 \text { min } \\
\text { over } 48 \text { weeks }\end{array}$ \\
\hline Weber et $a \beta^{\beta 3}$ & $\begin{array}{l}\text { Community, } \\
\text { psychotherapy } \\
\text { clinic, Netherlands }\end{array}$ & HIV & $\begin{array}{l}\text { Cognitive behavioural intervention } \\
\text { delivered by a psychotherapist }\end{array}$ & $\begin{array}{l}\text { Multiple components; } \\
\text { non-specific } \\
\text { techniques }\end{array}$ & $\begin{array}{l}\text { Standard care } \\
\text { (no details } \\
\text { provided) }\end{array}$ & 53 & $\begin{array}{l}\text { In person } \\
\text { (specialist) }\end{array}$ & $\begin{array}{l}11 \text { sessions of } \\
45 \text { min over } \\
12 \text { months }\end{array}$ \\
\hline Williams et $a^{\beta 1}$ & $\begin{array}{l}\text { Telephone calls } \\
\text { and visits to } \\
\text { patient's own } \\
\text { home, Australia }\end{array}$ & Diabetes & $\begin{array}{l}\text { Multifactorial intervention consisting } \\
\text { of self-monitoring of blood } \\
\text { pressure, medicine review, } \\
\text { educational DVDs and MI to } \\
\text { support blood pressure control and } \\
\text { optimal medication adherence }\end{array}$ & $\mathrm{Ml}$ & $\begin{array}{l}\text { Standard care } \\
\text { (no details } \\
\text { provided) }\end{array}$ & 75 & $\begin{array}{l}\text { In person and } \\
\text { phone calls } \\
\text { (specialist) }\end{array}$ & $\begin{array}{l}5 \text { sessions, } \\
\text { one of } 89 \text { min } \\
\text { and } 4 \text { of an } \\
\text { average of } \\
11.75 \text { min, } \\
\text { over } 3 \text { months }\end{array}$ \\
\hline
\end{tabular}

*See online supplementary table A for a detailed breakdown of intervention components. 
less, which was the case for 17 studies (65.4\%). Intervention exposure as the total number of minutes spent delivering the intervention could be estimated for 16 studies. In the remaining 10 studies, these data were not available. Intervention exposure ranged from $30 \mathrm{~min}$ to $8 \mathrm{~h}$ and $15 \mathrm{~min}$. The median (IQR) intervention exposure was 175 (118-263) min.

The comparison group was 'standard care' for all studies; for 13 studies $(50 \%)$, standard care involved some form of technique to improve adherence such as education, encouragement or provision of adherence aids and in these studies, recipients of the intervention received further techniques such as MI.

Supplementary figures S1 and S2 show the results of the risk of bias assessment. Only five (19.2\%) studies $^{27} 36414849$ scored 'low risk' in all five bias categories. Nineteen $(73.1 \%)$ studies were scored as moderate overall risk, because 2-4 of the categories were classed as 'low risk'. Two $(7.7 \%)^{40} 44$ studies were classed as 'high overall risk', because only one risk category was scored as low. The most common source of bias was a lack of blinding of the outcome assessment; this is because the measure of adherence was frequently selfreport. Self-report measures of adherence are commonly used but subject to patient bias. In the majority of cases, the patients were not blind to their treatment group allocation, and thus use of self-report measures leaves scope for bias.

\section{Meta-analysis}

Twenty-six RCTs were pooled to assess the effects of CBCTs on medication adherence. Three studies showed non-significant negative effects on medication adherence, but the remaining 23 studies all showed improvements in medication adherence with receipt of intervention. The effect size calculated for each study is summarised in table 2.

Random effects meta-analysis showed evidence that CBCTs are associated with improved medication adherence. Figure 2 shows the forest plot for the 26 studies and exemplifies the tendency towards positive adherence effects with intervention. A pooled estimate of effect size (95\% CI; reported as Hedges' g) of 0.34 (0.23 to 0.46$)$ was calculated when all studies were combined, although heterogeneity was high $\left(\mathrm{I}^{2}=68 \%, 95 \%\right.$ CI $52 \%$ to $79 \%)$.

The funnel plot produced was indicative of publication bias (as shown in figure 3) and thus further explored using Egger's test, which confirmed statistically significant funnel plot asymmetry $(p=0.005)$. The trim-and-fill technique was used to recompute an effect size which accounted for this asymmetry, yielding a more conservative effect size estimate of 0.21 (0.08 to 0.33; as shown in online supplementary figure S3). This effect size suggests that CBCTs elicit small but statistically significant improvements in medication adherence ( $p=0.001)$ relative to standard care. According to data from six studies that used the percentage of prescribed dose taken, the pooled SD of this outcome was $30.7 \%$. Then a standardised mean difference of 0.205 (0.084 to $0.326)$ will correspond to a difference of $6.3 \%$ (2.6\% to $10 \%)$ between the intervention and the control groups in the percentage of dose taken.

\section{Subgroup analyses via meta-regression}

Table 3 summarises the results of the subgroup analyses to explore variations in effect sizes for the predetermined variables. The regression coefficient is the difference in pooled Hedges' $g$ between the two subgroups compared. A coefficient $>0$ indicates that studies in subgroup-A reported greater treatment effects than those in subgroup-B.

The classification of studies into subgroups was largely intuitive. However, as a continuous rather than a categorical variable, 'total intervention exposure' was less amenable to intuitive dichotomisation. In such instances, it is standard practice to create two subgroups by distributing a roughly equal number of studies to each group. An arbitrary cut-off point of $3 \mathrm{~h}$ was therefore used to split the data into two subgroups.

Interventions delivered from hospital settings were associated with greater treatment effects compared with interventions in community or other settings (difference $0.27,95 \%$ CI 0.01 to $0.54, \mathrm{p}=0.043$ ). Differences in effect sizes between subgroups were statistically non-significant in all other cases. However, the subgroup analyses may have failed to detect important differences between subgroups because of the small number of studies included.

As the variable 'intervention exposure' was a continuous variable, an additional post hoc analysis was undertaken. This allowed the variable to be analysed in its 'natural' continuous state rather than in two subgroups. This exploratory analysis was undertaken to ensure that the arbitrary cut-off point of $3 \mathrm{~h}$ had not adversely influenced the data. A coefficient value $(95 \%$ CI) of 0.001 $(-0.001$ to 0.002$)$ suggested that there was no association between intervention exposure and effect size. A non-significant $\mathrm{p}$ value of 0.540 confirmed this and demonstrates results comparable to the subgroup analysis for this variable.

\section{DISCUSSION}

\section{Principal findings}

Receipt of a cognitive-based behavioural adherence intervention was associated with small but statistically significant improvements in medication adherence. Heterogeneity was high and notable publication bias was identified. However, techniques have been used to account for this bias, resulting in a more conservative summary effect size of $0.21 \quad(95 \%$ CI 0.08 to 0.33 ; $\mathrm{p}=0.001$ ).

In half of the included studies, the standard care received by the control group explicitly involved some form of 'adherence enhancing strategy' such as provision of education, monitoring or review. Such strategies 


\begin{tabular}{|c|c|c|c|c|c|c|}
\hline \multirow[b]{2}{*}{ Study } & \multirow{2}{*}{$\begin{array}{l}\text { Sample size } \\
\text { (intervention, control) }\end{array}$} & \multirow[b]{2}{*}{ Adherence definition (assessment measure) } & \multicolumn{3}{|l|}{ Extracted data } & \multirow{2}{*}{$\begin{array}{l}\text { Effect size } \\
\text { (Hedges' g) } \\
(95 \% \mathrm{Cl})\end{array}$} \\
\hline & & & $\begin{array}{l}\text { Intervention } \\
\text { group }\end{array}$ & Control group & p Value & \\
\hline Bailey et $a \beta^{34}$ & $225(124,101)$ & $\begin{array}{l}\text { Percentage of patients scored as adherent on all } \\
6 \text { items of a self-report scale (based on Morisky's } \\
\text { self-reported scale) }\end{array}$ & Mean=91.9 & Mean $=61.7$ & 0.001 & $0.44(0.18$ to 0.71$)$ \\
\hline Berger et $a{ }^{40}$ & $367(172,195)$ & $\begin{array}{l}\text { Percentage of patients discontinuing treatment by } \\
\text { study endpoint (patient interview) }\end{array}$ & Mean $=98.8$ & Mean $=91.3$ & 0.001 & $0.35(0.14$ to 0.55$)$ \\
\hline Brown et $a f^{29}$ & $69(36,33)$ & $\begin{array}{l}\text { Percentage of prescribed doses taken over a } \\
\text { month (electronic monitoring) }\end{array}$ & $\begin{array}{l}\text { Mean }(S D)=93.4 \\
(12.3)\end{array}$ & $\begin{array}{l}\text { Mean }(\mathrm{SD}) \\
=79.1(28.1)\end{array}$ & & $0.66(0.18$ to 1.14$)$ \\
\hline Dilorio et $a{ }^{41}$ & $17(8,9)$ & $\begin{array}{l}\text { Mean number of missed medicines in the last } \\
30 \text { days (self-report questionnaire) }\end{array}$ & $\begin{array}{l}\text { Mean }(S D)=0.13 \\
(0.35)\end{array}$ & $\begin{array}{l}\text { Mean }(\mathrm{SD}) \\
=0.98(1.48)\end{array}$ & & $0.73(-0.21$ to 1.67$)$ \\
\hline Dilorio et $a{ }^{42}$ & $213(107,106)$ & $\begin{array}{l}\text { Percentage of doses taken during the } \\
\text { intervention period (electronic monitoring) }\end{array}$ & Mean $=64$ & Mean $=55$ & 0.09 & $0.23(-0.04$ to 0.50$)$ \\
\hline Farmer et $a^{R^{7}}$ & $211(126,85)$ & $\begin{array}{l}\text { Percentage of days during a } 12 \text {-week period in } \\
\text { which medication was taken correctly (electronic } \\
\text { monitoring) }\end{array}$ & $\begin{array}{l}\text { Mean }(\mathrm{SD})=77.4 \\
(26.3)\end{array}$ & $\begin{array}{l}\text { Mean (SD) } \\
=64.0(30.8)\end{array}$ & 0.04 & $0.47(0.20$ to 0.75$)$ \\
\hline George et $a \beta^{\beta 0}$ & $343(170,173)$ & $\begin{array}{l}\text { Percentage of participants classed as adherent } \\
\text { (Morisky self-report scale) }\end{array}$ & Mean $=72.2$ & Mean $=63.8$ & 0.09 & $0.18(-0.03$ to 0.39$)$ \\
\hline Golin et $a^{\beta 9}$ & $117(59,58)$ & $\begin{array}{l}\text { Percentage of prescribed doses taken in the } \\
\text { month prior to the study endpoint (CAS) }\end{array}$ & $\begin{array}{l}\text { Mean }(S D)=76 \\
(27)\end{array}$ & $\begin{array}{l}\text { Mean }(S D)=71 \\
(27)\end{array}$ & & $0.18(-0.18$ to 0.54$)$ \\
\hline Hovell et $a^{p_{1}^{1}}$ & $188(92,96)$ & $\begin{array}{l}\text { Cumulative number of doses taken over } \\
9 \text { months (patient interview) }\end{array}$ & $\begin{array}{l}\text { Mean }(S D) \\
=179.93(57.01)\end{array}$ & $\begin{array}{l}\text { Mean }(\mathrm{SD}) \\
=150.98(73.75)\end{array}$ & & $0.44(0.15$ to 0.72$)$ \\
\hline $\begin{array}{l}\text { Konkle-Parker et al } \\
38\end{array}$ & $36(21,15)$ & $\begin{array}{l}\text { Percentage of patients taking }>90 \% \text { of their } \\
\text { medications in the last } 3-4 \text { weeks (prescription } \\
\text { refill data) }\end{array}$ & $\begin{array}{l}\text { Mean }(S D)=0.93 \\
(0.23)\end{array}$ & $\begin{array}{l}\text { Mean }(\mathrm{SD}) \\
=0.92(0.27)\end{array}$ & & $0.04(-0.61$ to 0.69$)$ \\
\hline $\begin{array}{l}\text { Maneesriwongul } \\
\text { et } a l^{\beta 7}\end{array}$ & $60(30,30)$ & $\begin{array}{l}\text { Mean percentage of doses taken over the last } \\
4 \text { weeks (self-report using a visual analogue } \\
\text { scale) }\end{array}$ & $\begin{array}{l}\text { Mean }(S D)=97.1 \\
(3.3)\end{array}$ & $\begin{array}{l}\text { Mean }(\mathrm{SD}) \\
=89.8(5.6)\end{array}$ & & 1.55 (0.98 to 2.12$)$ \\
\hline Murphy et $a F^{2}$ & $33(17,16)$ & $\begin{array}{l}\text { Percentage of doses taken during the } \\
\text { intervention period (self-report questionnaire) }\end{array}$ & $\begin{array}{l}\text { Mean }(S D)=0.86 \\
(0.33)\end{array}$ & $\begin{array}{l}\text { Mean }(S D) \\
=0.83(0.36)\end{array}$ & & $0.09(-0.58$ to 0.75$)$ \\
\hline Ogedegbe et $a^{\beta 6}$ & $160(79,81)$ & $\begin{array}{l}\text { Percentage of days during a 2-month period in } \\
\text { which medication was taken correctly (electronic } \\
\text { monitoring) }\end{array}$ & Mean $=56.9$ & Mean $=42.9$ & 0.027 & 0.35 (0.04 to 0.66$)$ \\
\hline Pradier et $a^{50}$ & $202(123,121)$ & $\begin{array}{l}\text { Percentage of patients deemed to be adherent } \\
\text { (taking } 100 \% \text { of doses; self-report questionnaire) }\end{array}$ & Mean $=75$ & Mean $=61$ & 0.04 & 0.34 (0.02 to 0.65$)$ \\
\hline Put et $a^{\beta 5}$ & $23(12,11)$ & $\begin{array}{l}\text { Frequency of non-adherent behaviour over the } \\
\text { last } 3 \text { months (self-report questionnaire) }\end{array}$ & $\begin{array}{l}\text { Mean }(S D)=6.9 \\
(1.2)\end{array}$ & $\begin{array}{l}\text { Mean }(\mathrm{SD})=8.1 \\
(3.1)\end{array}$ & & $0.50(-0.30$ to 1.30$)$ \\
\hline Remien et $a f^{49}$ & $196(106,109)$ & $\begin{array}{l}\text { Percentage of doses taken during the previous } \\
2 \text { weeks (electronic monitoring) }\end{array}$ & $\begin{array}{l}\text { Mean }(S D)=76 \\
(27)\end{array}$ & $\begin{array}{l}\text { Mean }(S D)=60 \\
(34)\end{array}$ & & 0.52 (0.25 to 0.79$)$ \\
\hline Safren et $a{ }^{44}$ & $53(28,25)$ & $\begin{array}{l}\text { Percentage of prescribed doses taken over the } \\
\text { last } 2 \text { weeks (self-report questionnaire) }\end{array}$ & $\begin{array}{l}\text { Mean }(S D)=93 \\
(22)\end{array}$ & $\begin{array}{l}\text { Mean }(S D)=94 \\
(10)\end{array}$ & & $-0.06(-0.59$ to 0.47$)$ \\
\hline
\end{tabular}


Table 2 Continued

\begin{tabular}{|c|c|c|c|c|c|c|}
\hline \multirow[b]{2}{*}{ Study } & \multirow[b]{2}{*}{$\begin{array}{l}\text { Sample size } \\
\text { (intervention, control) }\end{array}$} & \multirow[b]{2}{*}{ Adherence definition (assessment measure) } & \multicolumn{3}{|l|}{ Extracted data } & \multirow{2}{*}{$\begin{array}{l}\text { Effect size } \\
\text { (Hedges' g) } \\
(95 \% \mathrm{Cl})\end{array}$} \\
\hline & & & $\begin{array}{l}\text { Intervention } \\
\text { group }\end{array}$ & Control group & p Value & \\
\hline $\begin{array}{l}\text { Sheeran and } \\
\text { Orbell }^{28}\end{array}$ & $78(38,40)$ & $\begin{array}{l}\text { Number of once daily doses missed over a } \\
3 \text { week period (self-report questionnaire) }\end{array}$ & Mean $=2.68$ & Mean $=4.85$ & 0.05 & $0.45(0.00$ to 0.89$)$ \\
\hline Simoni et $a f^{48}$ & $114(57,57)$ & $\begin{array}{l}\text { Percentage of doses taken over the last } 7 \text { days } \\
\text { (electronic monitoring) }\end{array}$ & $\begin{array}{l}\text { Mean }(\mathrm{SD})=32.3 \\
(42.5)\end{array}$ & $\begin{array}{l}\text { Mean (SD) } \\
=29.1(39.7)\end{array}$ & & $0.08(-0.29$ to 0.44$)$ \\
\hline Smith et $a f^{47}$ & $17(8,9)$ & $\begin{array}{l}\text { Percentage of participants taking } \geq 80 \% \text { of their } \\
\text { weekly doses (electronic monitoring) }\end{array}$ & \multicolumn{2}{|c|}{$\mathrm{OR}=7.8(2.2$ to 28.1$)$} & & $1.08(0.41$ to 1.74$)$ \\
\hline Solomon et $a{ }^{43}$ & $2087(1046,1041)$ & $\begin{array}{l}\text { Median \% medication possession ratio } \\
\text { (prescription refill data) }\end{array}$ & $\begin{array}{l}\text { Median }=49 \\
\mathrm{IQR}=7 \text { to } 88\end{array}$ & $\begin{array}{l}\text { Median }=41 \\
\mathrm{IQR}=2 \text { to } 86\end{array}$ & 0.07 & $0.08(-0.01$ to 0.17$)$ \\
\hline Tuldrà et $a{ }^{46}$ & $77(36,41)$ & $\begin{array}{l}\text { Percentage of patients with monthly adherence } \\
\geq 95 \% \text { (self-reported number of pills taken) }\end{array}$ & Mean $=94$ & Mean $=69$ & 0.008 & $0.62(0.16$ to 1.07$)$ \\
\hline van Es et $a \beta^{\beta 2}$ & $67(58,54)$ & $\begin{array}{l}\text { Adherence score on a self-report scale based on } \\
\text { how often medication was taken (never-always) }\end{array}$ & Mean $=7.7$ & Mean $=6.7$ & 0.05 & 0.48 (0.00 to 0.96$)$ \\
\hline Wagner et $a{ }^{45}$ & $135(154,76)$ & $\begin{array}{l}\text { Percentage of doses taken during the } \\
\text { intervention period (electronic monitoring) }\end{array}$ & Mean $=83.5$ & Mean $=86.4$ & 0.57 & $-0.08(-0.35$ to 0.20$)$ \\
\hline Weber et $a \beta^{33}$ & $53(29,24)$ & $\begin{array}{l}\text { Percentage of patients with monthly adherence } \\
\geq 95 \% \text { (electronic monitoring) }\end{array}$ & Mean $=70.8$ & Mean $=50$ & 0.014 & 0.69 (0.14 to 1.24$)$ \\
\hline Williams et $a \beta^{31}$ & $75(36,39)$ & $\begin{array}{l}\text { Percentage of doses taken during the } \\
\text { intervention period (pill counts }\end{array}$ & Mean $=58.4$ & Mean $=66$ & 0.162 & $-0.32(-0.77$ to 0.13$)$ \\
\hline
\end{tabular}




\section{Study}

ID

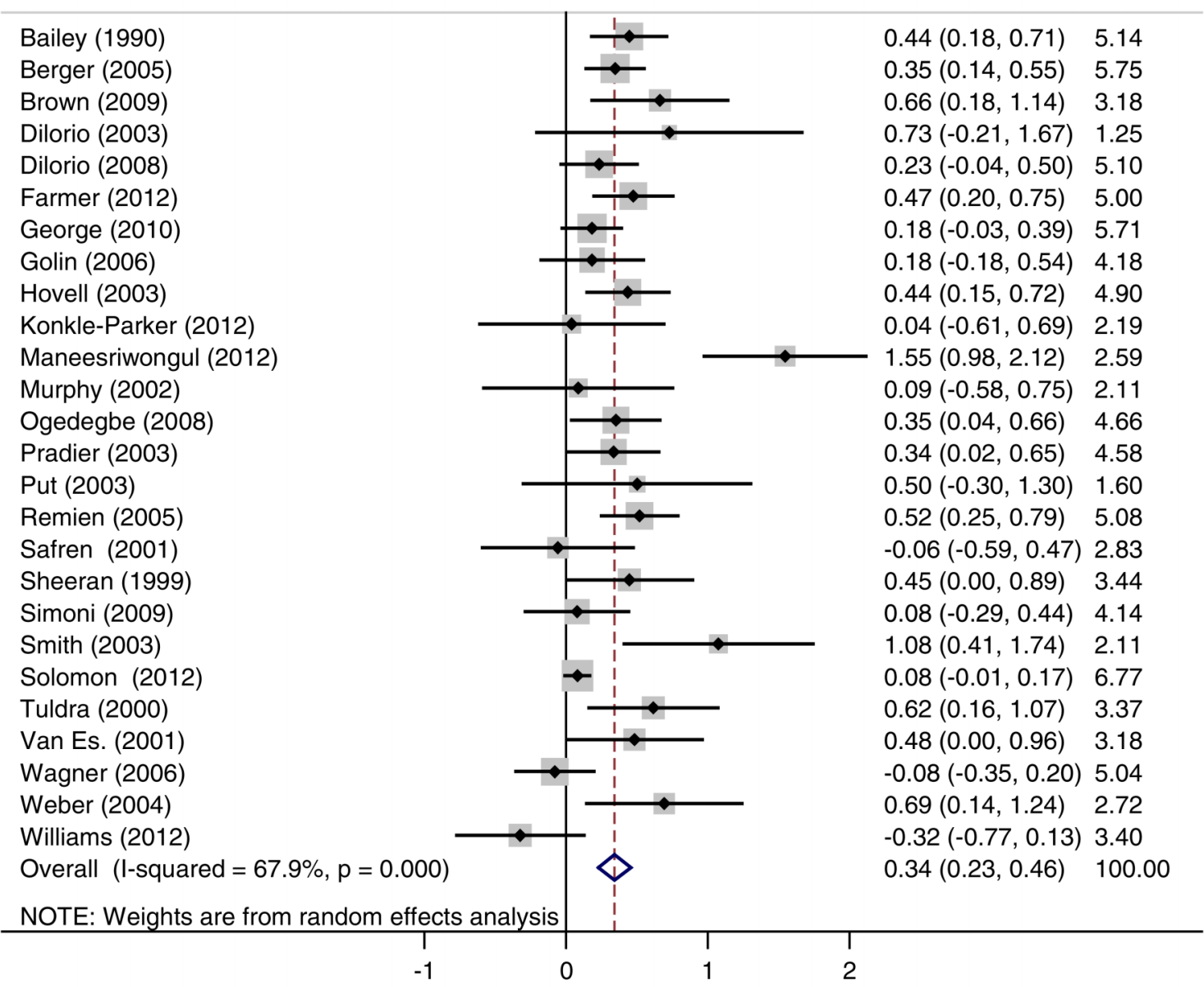

Favours control

Figure 2 Forrest plot for studies included in meta-analysis.

Favours intervention

\section{Hedges' \\ g (95\% Cl) \\ $\%$ \\ Weight}

form the mainstay of current medication adherence interventions, and so our research suggests that CBCTs may be able to elicit adherence benefits beyond the techniques used in current practice.

The majority of interventions were complex and multifaceted, and thus subgroup analysis to explore whether this is associated with greater effect could not be undertaken. The subgroup analyses performed revealed that the effect size was greater when interventions were delivered in a hospital setting than in a community setting, but were not influenced by other variables such as the type of CBCT, delivery method and personnel or duration. Further work is necessary to explore the effect of settings on effect size.

\section{Comparison with other studies}

In 2003, Peterson et $a e^{53}$ conducted a meta-analysis of educational and behavioural interventions to improve medication adherence in a range of illnesses. The included studies were all RCTs delivered over similar time periods to those included in our study. The educational components and behavioural components such as changes in dosing schedule and reminders examined by Peterson et al closely mirror those utilised in the studies from our meta-analysis which used control groups with 'active standard care'. Peterson et al reported a correlation coefficient ( $r$ ) equivalent to a Cohen's d effect size of $0.16(0.08,0.24)$. For our study, the effect size for all studies, when adjusting for publication bias and reported as Hedges' g, was 0.20 (0.08, 0.33). This suggests that inclusion of CBCTs strengthens the adherence improvements gained, if only marginally. Moreover, Peterson et al report publication bias observed from a funnel plot of their included studies, but have not made allowances for this bias via recomputed effect sizes. Their Cohen's $d$ value of 0.16 is quite likely exaggerated by the noted publication bias and thus implies that the true difference in effect size between the two meta-analyses may be greater.

An effect size (Hedges' g) of 0.25 (95\% CI 0.07 to 0.42 ) for studies using MI was calculated, compared with an effect size of 0.41 (95\% CI 0.278 to 0.541$)$ for non-MI interventions. After adjusting for bias, the estimated Hedges' g was 0.137 (95\% CI -0.067 to 0.341$)$ 
Figure 3 Funnel plot for studies included in meta-analysis.

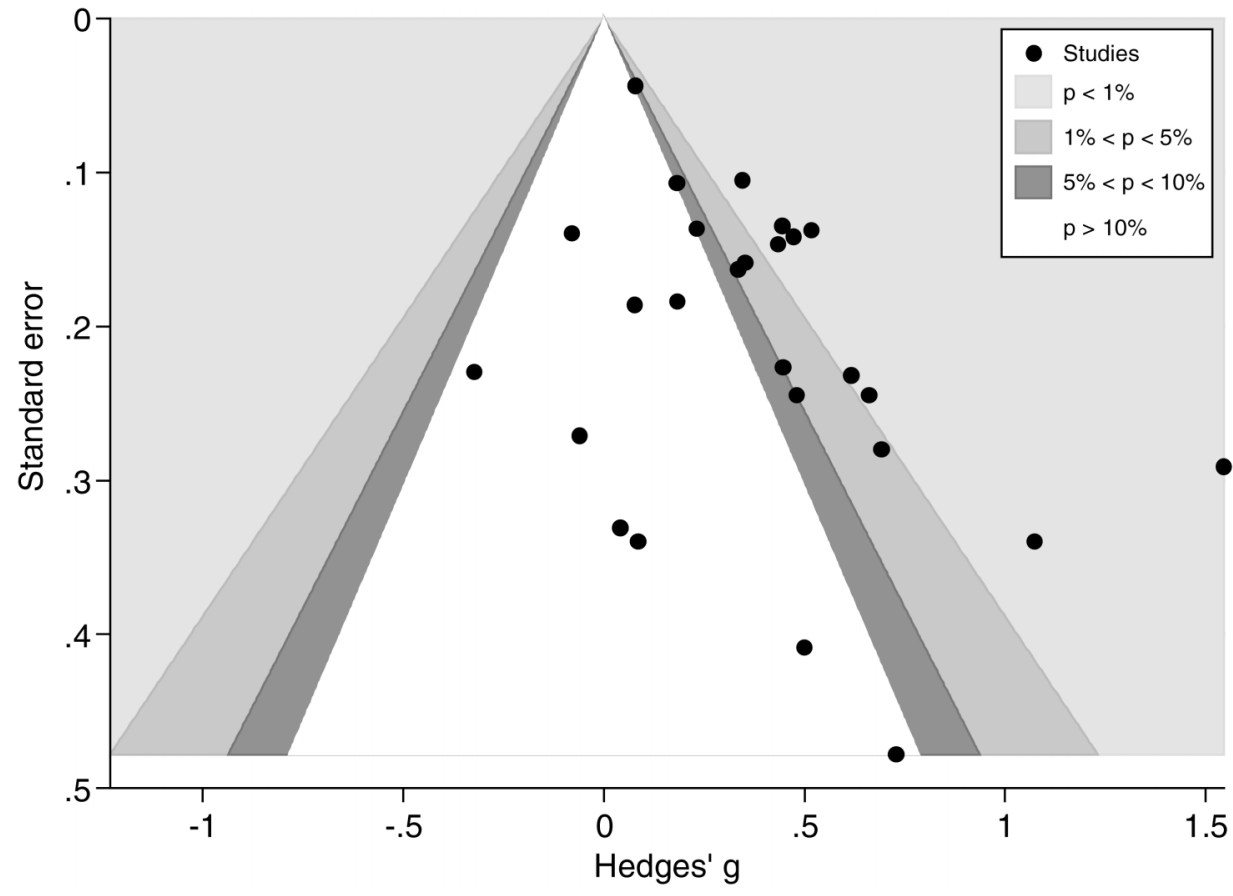

for studies using MI and 0.356 (95\% CI 0.223 to 0.489 ) for studies using non-MI interventions. These estimated effect sizes closely match the effect size calculated when MI is used as a behavioural intervention in other healthcare domains ${ }^{14}$ and thus represent novel evidence for the wider application of MI techniques beyond the treatment of substance abuse and gambling. The overlapping CIs of the effect sizes calculated for MI-based and non-MI based interventions suggest that MI-based interventions are unlikely to be superior

Table 3 Summary of subgroup analyses

\begin{tabular}{|c|c|c|c|c|}
\hline Variable & Subgroup-A vs subgroup-B & $\begin{array}{l}\text { Number of studies (number of } \\
\text { participants) in each subgroup }\end{array}$ & Coefficient $(95 \% \mathrm{Cl})$ & $\begin{array}{l}\mathbf{p} \\
\text { Value }\end{array}$ \\
\hline $\begin{array}{l}\text { Intervention } \\
\text { setting }\end{array}$ & Hospital vs community & 9 (1124) vs 17 (4092) & $0.27(0.01$ to 0.54$)$ & 0.043 \\
\hline Disease area & HIV vs other conditions & 14 (1323) vs 12 (3893) & $0.05(-0.23$ to 0.33$)$ & 0.72 \\
\hline $\begin{array}{l}\text { Intervention } \\
\text { components }\end{array}$ & MI vs no MI component & 11 (3538) vs 15 (1678) & $-0.17(-0.44$ to 0.09$)$ & 0.193 \\
\hline \multirow[t]{3}{*}{$\begin{array}{l}\text { Intervention } \\
\text { delivery method }\end{array}$} & $\begin{array}{l}\text { Entirely in person vs other } \\
\text { methods }\end{array}$ & 15 (1663) vs 11 (3553) & $-0.03(-0.31$ to 0.25$)$ & 0.841 \\
\hline & $\begin{array}{l}\text { Entirely over the telephone vs } \\
\text { other methods }\end{array}$ & 3 (2679) vs 23 (2537) & $-0.16(-0.59$ to 0.26$)$ & 0.442 \\
\hline & $\begin{array}{l}\text { Both in person and telephone vs } \\
\text { other }\end{array}$ & $7(775)$ vs 19 (4441) & $-0.05(-0.27$ to 0.37$)$ & 0.744 \\
\hline $\begin{array}{l}\text { Intervention } \\
\text { delivery personnel }\end{array}$ & Specialist vs Routine HCP & 5 (503) vs 12 (1567) & $-0.01(-0.46$ to 0.26$)$ & 0.561 \\
\hline $\begin{array}{l}\text { Total intervention } \\
\text { exposure }\end{array}$ & $\leq 3 h$ vs $>3 h$ & 9 (3061) vs 7 (887) & $0.07(-0.35$ to 0.50$)$ & 0.728 \\
\hline $\begin{array}{l}\text { Control group } \\
\text { type }\end{array}$ & $\begin{array}{l}\text { Explicit active controls vs usual } \\
\text { care (no adherence enhancing } \\
\text { strategies) }\end{array}$ & 13 (3683) vs 13 (1533) & $0.09(-0.18$ to 0.37$)$ & 0.493 \\
\hline Risk of bias & $\begin{array}{l}\text { Outcome assessment blinding } \\
\text { vs no outcome assessment } \\
\text { blinding }\end{array}$ & 15 (3555) vs 11 (1661) & $0.05(-0.24$ to 0.33$)$ & 0.736 \\
\hline $\begin{array}{l}\text { Outcome } \\
\text { measures }\end{array}$ & $\begin{array}{l}\text { Objective vs subjective } \\
\text { measured outcomes }\end{array}$ & $14(3850)$ vs 12 (1366) & $-0.16(-0.44$ to 0.11$)$ & 0.225 \\
\hline
\end{tabular}


in their efficacy compared with those based on other CBCTs.

\section{Strengths and weaknesses of our work}

This study represents the first meta-analysis of MI and other CBCTs as medication adherence interventions and has been undertaken with methodological rigour and in accordance with published guidance. ${ }^{18}$ A notable strength of this work is the robust methodological techniques that have been applied to provide an estimate of effect size which accounts for publication biases, and thus greater confidence can be placed in the estimate. The work is also strengthened by restriction to RCTs.

While moderate agreement in abstract screening may be lower than ideal, this is largely attributable to the paucity of detail reported in abstracts and complexities in intervention definitions, which are known to be problematic in this domain. ${ }^{11-13}$ The conservative approach to abstract screening prevented study exclusion if disagreement was associated with insufficient information and thus prevented exclusion in error. Heterogeneity between the included studies was high with an $\mathrm{I}^{2}$ value of $68 \%$ (95\% CI $52 \%$ to $79 \%$ ) and thus raises the question as to whether the studies were sufficiently comparable to warrant pooling in a meta-analysis. While we defined our inclusion criteria to ensure that studies were as similar as possible (ie, all using a CBCT), heterogeneity was expected as other factors such as the populations and disease states studied were more difficult to control for. Interestingly, the largest study had a small standardised group difference compared with most of the other studies which contributed substantially to the heterogeneity. ${ }^{43}$ Aside from these between-study differences, the actual interventions were variable, as were the definitions of adherence and assessment tools used.

The differences between subgroups were statistically non-significant in terms of disease area, intervention components, delivery methods, delivery personnel, intensity, usual care and risk of bias. However, the statistical power was limited by the small number of studies included in the subgroup analyses. The analyses may therefore have failed to detect some important subgroup differences. Moreover, for variables such as the intervention exposure, meaningful conclusions are difficult to draw. While the analyses infer that intervention exposure did not influence effect size, it is important to remember that a whole host of variables are at large. It is possible that briefer interventions used different techniques or were delivered to different types of recipients compared with longer interventions, and therefore comparisons may not be wholly meaningful. Further work may be necessary to explore whether otherwise identical interventions (same technique, same population, same delivery personnel and so forth) differ in effect size when delivered with different exposure.

Despite these numerous between-study differences, the core of each intervention was the use of a CBCT to improve medication adherence, which was comparable across all studies, and thus we would argue that data pooling irrespective of heterogeneity was both intuitive and meaningful.

We have established that receipt of a cognitive-based behavioural medication adherence intervention is likely to elicit small improvements in medication adherence, but the clinical relevance and impact of this improvement remain unknown. Based on the mean adherence rates in the control groups, mean SDs and the effect size calculated, it has been possible to estimate the increase in percentage of doses taken for the intervention groups. Based on the adjusted Hedges' g value of 0.205 (0.084 to 0.326), receipt of a CBCT improved adherence (\% of doses taken) by $6.29 \%$ (2.58\% to $10 \%)$. For some medications, a $6 \%$ increase in the percentage of doses taken may not be of clinical relevance. However, for other medications such as antiretroviral therapy for HIV, which requires very high levels of adherence or antiepileptic therapies with narrow therapeutic windows, a $6 \%$ increase in adherence may have notable clinical relevance. While many included studies provided data on clinical outcomes, pooling of these data from a diverse range of studies was not possible.

\section{Implications}

Motivational and CBCTs can seemingly be delivered effectively by routine healthcare professionals, with efficacy applicable to a range of diseases. Efficacy was not related to intervention exposure. Interestingly, the results also suggest that these interventions can be delivered via telephone or face-to-face with comparable efficacy. These are valuable traits for an adherence intervention which could be adaptable to a wide range of settings and amenable to tailoring to meet individual needs.

The flexibility and adaptability of these techniques coupled with their frequent simplicity means that practitioners may wish to consider incorporation of these techniques into their consultations when faced with the need to facilitate medication-related behaviour changes.

\section{RECOMMENDATIONS AND CONCLUSIONS}

Further investigation of these techniques as medication adherence interventions is warranted in order to further elucidate the characteristics most strongly associated with efficacy. Studies to determine patient and healthcare practitioner acceptability of these techniques are also necessary to establish their role in routine healthcare.

Acknowledgements CE would like to acknowledge Estelle Payerne for her invaluable contributions as the second reviewer for abstract screening and data extraction. CE would also like to acknowledge David Wright and John Wood for their comments on the protocol design, data analysis and methods of dissemination, and Steven Watson for his ongoing technical support in using the meta-analysis software and comments regarding data interpretation.

Contributors DB and CE were responsible for the overall study co-ordination. CE was responsible for the study conception and protocol design, under the supervision of DB with contributions from FS. All literature searching, abstract screening, study selection and data extraction was undertaken independently 
by CE and EP with referral to DB as a third reviewer as necessary.

Assessment of methodological quality was also undertaken by CE and EP. CE was responsible for all data analysis with guidance from DB and FS. Statistical tests, asymmetry tests and trim and fill methods were undertaken by FS. CE wrote the first draft of the paper with guidance from DB and advice from FS. All authors have read and approved the final version of the manuscript.

Funding This research has been supported by a PhD studentship awarded by the Dean of Science at the University of East Anglia.

\section{Competing interests None.}

Provenance and peer review Not commissioned; externally peer reviewed.

Data sharing statement No additional data are available.

Open Access This is an Open Access article distributed in accordance with the Creative Commons Attribution Non Commercial (CC BY-NC 3.0) license, which permits others to distribute, remix, adapt, build upon this work noncommercially, and license their derivative works on different terms, provided the original work is properly cited and the use is non-commercial. See: http:// creativecommons.org/licenses/by-nc/3.0/

\section{REFERENCES}

1. World Health Organisation. Adherence to long term therapies: evidence for action. Geneva: World Health Organisation, 2003.

2. The National Collaborating Centre for Primary Care. The National Institute for Health and Clinical Excellence (NICE) clinical guideline 76, full guidance. Medicines adherence; involving patients in decisions about prescribed medicines and supporting adherence. London: The National Institute for Health and Clinical Excellence, 2009.

3. Simpson SH, Eurich DT, Majumdar SR. A meta-analysis of the association between adherence to drug therapy and mortality. BMJ 2006;333:15.

4. Haynes RB, Ackloo E, Sahota N, et al. Interventions for enhancing medication adherence. Cochrane Database Syst Rev 2008;2: CD000011. doi:10.1002/14651858.CD000011.pub3.

5. Clifford S, Garfield S, Eliasson L, et al. Medication adherence and community pharmacy: a review of education, policy and research in England. Pharm Pract (Internet) 2010;8:77-88.

6. Horne R, Weinman J, Barber N, et al. Concordance, adherence and compliance in medicine taking. London: National Co-ordinating Centre for NHS Service Delivery and Organisation Research \& Development; 2005, Report for the National Co-ordinating Centre for NHS Service Delivery and Organisation R \& D (NCCSDO).

7. Britt E, Hudson SM, Blampied NM. Motivational interviewing in health settings: a review. Patient Educ Counsel 2004;53:147-55

8. Miller WR, Rollnick SR. Motivational interviewing: preparing people to change behaviour. New York: Guilford Press, 1991.

9. Miller WR, Rollnick S. Motivational interviewing: preparing people for change. The Guilford Press, 2002.

10. Dunn C, Deroo L, Rivara FP. The use of brief interventions adapted from motivational interviewing across behavioral domains: a systematic review. Addiction 2001;96:1725-42.

11. Knight KM, McGowan L, Dickens C, et al. A systematic review of motivational interviewing in physical health care settings. $\mathrm{Br} \mathrm{J}$ Health Psychol 2006;11:319-32.

12. Burke BL, Arkowitz H, Menchola M. The efficacy of motivational interviewing: a meta-analysis of controlled clinical trials. J Consult Clin Psychol 2003;71:843.

13. Rubak S, Sandbæk A, Lauritzen T, et al. Motivational interviewing: a systematic review and meta-analysis. Br J Gen Pract 2005;55:305.

14. Hettema J, Steele J, Miller WR. Motivational interviewing. Annu Rev Clin Psychol 2005;1:91-111.

15. Lundahl B, Burke BL. The effectiveness and applicability of motivational interviewing: a practice friendly review of four meta analyses. J Clin Psychol 2009;65:1232-45.

16. Lundahl BW, Kunz C, Brownell C, et al. A meta-analysis of motivational interviewing: twenty-five years of empirical studies. Res Soc Work Pract 2010;20:137.

17. Rollnick S, Allison J, Ballasiotes S, et al. Variations on a theme: motivational interviewing and its adaptations. Motivational interviewing: preparing people for change. 2nd edition. New York, 2002:270-83

18. Higgins JPT, Green S, eds. Cochrane Handbook for Systematic Reviews of Interventions Version 5.1.0 [updated March 2011]. The Cochrane Collaboration, 2011. Available from www.cochranehandbook.org (accessed 4 Jul 2011).
19. Moher D, Liberati A, Tetzlaff J, et al. Preferred reporting items for systematic reviews and meta-analyses: the PRISMA statement. BMJ 2009;339:332-6.

20. Landis JR, Koch GG. The measurement of observer agreement for categorical data. Biometrics 1977:159-74

21. Higgins J, Thompson SG. Quantifying heterogeneity in a meta analysis. Stat Med 2002;21:1539-58.

22. Higgins $\mathrm{J}$, Thompson S, Deeks $\mathrm{J}$, et al. Measuring inconsistency in meta-analysis. BMJ 2003;327:557-60.

23. Borenstein M, Hedges LV, Higgins JPT, et al. Introduction to meta-analysis. Wiley Online Library, 2009.

24. Egger M, Smith GD, Schneider M, et al. Bias in meta-analysis detected by a simple, graphical test. BMJ 1997;315:629-34.

25. Duval S, Tweedie R. A nonparametric "trim and fill" method of accounting for publication bias in meta-analysis. J Am Stat Assoc 2000;95:89-98.

26. Duval S, Tweedie R. Trim and fill: a simple funnel-plot-based method of testing and adjusting for publication bias in meta-analysis. Biometrics 2000;56:455-63.

27. Farmer A, Hardeman W, Hughes D, et al. An explanatory randomised controlled trial of a nurse-led, consultation-based intervention to support patients with adherence to taking glucose lowering medication for type 2 diabetes. BMC Fam Pract 2012;13:30-8

28. Sheeran $\mathrm{P}$, Orbell $\mathrm{S}$. Implementation intentions and repeated behaviour: augmenting the predictive validity of the theory of planned behaviour. Eur J Soc Psychol 1999;29:349-69.

29. Brown I, Sheeran P, Reuber M. Enhancing antiepileptic drug adherence: a randomized controlled trial. Epilepsy Behav 2009;16:634-9.

30. George J, McNamara K, Jackson S, et al. The HAPPY trial: a randomised controlled trial of a community pharmacy-based intervention for improving patient adherence to antihypertensive medicines. Int J Pharm Pract 2010;Conference: 2010 Royal Pharmaceutical Society Conference London United Kingdom. Conference Start: 20100905 Conference End: 20100906. Conference Publication: (var.pagings). 18:22-3.

31. Williams A, Manias E, Walker R, et al. A multifactorial intervention to improve blood pressure control in co-existing diabetes and kidney disease: a feasibility randomized controlled trial. J Adv Nur 2012;68:2515-25.

32. van Es SM, Nagelkerke AF, Colland VT, et al. An intervention programme using the ASE-model aimed at enhancing adherence in adolescents with asthma. Patient Educ Couns 2001;44:193-203.

33. Weber R, Christen L, Christen S, et al. Effect of individual cognitive behaviour intervention on adherence to antiretroviral therapy: prospective randomized trial. Antivir Ther 2004;9:85-96.

34. Bailey WC, Richards JM Jr, Brooks CM, et al. A randomized trial to improve self-management practices of adults with asthma. Arch Intern Med 1990;150:1664-8.

35. Put $\mathrm{C}$, van den Bergh $\mathrm{O}$, Lemaigre $\mathrm{V}$, et al. Evaluation of an individualised asthma programme directed at behavioural change. Eur Respir J 2003;21:109-15.

36. Ogedegbe G, Chaplin W, Schoenthaler A, et al. A practice-based trial of motivational interviewing and adherence in hypertensive African Americans. Am J Hypertens 2008;21:1137-43.

37. Maneesriwongul W, Prajanket O-O, Saengcharnchai P. Effects of motivational interviewing or an educational video on knowledge about HIV/AIDS, health beliefs and antiretroviral medication adherence among adult Thais with HIV/AIDS. Pac Rim Int J Nurs Res 2012;16:124-37.

38. Konkle-Parker DJ, Erlen JA, Dubbert PM, et al. Pilot testing of an HIV medication adherence intervention in a public clinic in the Deep South. J Am Acad Nurse Pract 2012;24:488-98.

39. Golin CE, Earp J, Tien H-C, et al. A 2-arm, randomized, controlled trial of a motivational interviewing-based intervention to improve adherence to antiretroviral therapy (ART) among patients failing or initiating ART. JAIDS 2006;42:42-51.

40. Berger BA, Liang $\mathrm{H}$, Hudmon KS. Evaluation of software-based telephone counseling to enhance medication persistency among patients with multiple sclerosis. J Am Pharm Assoc 2005;45:466-72.

41. Dilorio C, Resnicow K, McDonnell M, et al. Using motivational interviewing to promote adherence to antiretroviral medications: a pilot study. J Assoc Nurses AIDS Care 2003;14:52-62.

42. Dilorio C, McCarty F, Resnicow K, et al. Using motivational interviewing to promote adherence to antiretroviral medications: a randomized controlled study. AIDS Care 2008;20:273-83.

43. Solomon $\mathrm{DH}$, Iversen MD, Avorn J, et al. Osteoporosis telephonic intervention to improve medication regimen adherence: a large, pragmatic, randomized controlled trial. Arch Intern Med 2012;172:477-83. 
44. Safren SA, Otto MW, Worth JL, et al. Two strategies to increase adherence to HIV antiretroviral medication: life-steps and medication monitoring. Behav Res Ther 2001;39:1151-62.

45. Wagner GJ, Kanouse DE, Golinelli D, et al. Cognitive-behavioral intervention to enhance adherence to antiretroviral therapy: a randomized controlled trial (CCTG 578). AIDS 2006;20:1295-302.

46. Tuldrà $\mathrm{A}$, Fumaz $\mathrm{CR}$, Ferrer $\mathrm{MJ}$, et al. Prospective randomized two-arm controlled study to determine the efficacy of a specific intervention to improve long-term adherence to highly active antiretroviral therapy. JAIDS 2000;25:221-8.

47. Smith SR, Rublein JC, Marcus C, et al. A medication self-management program to improve adherence to HIV therapy regimens. Patient Educ Couns 2003;50:187-99.

48. Simoni JM, Huh D, Frick PA, et al. Peer support and pager messaging to promote antiretroviral modifying therapy in Seattle: a randomized controlled trial. JAIDS 2009;52:465-73.
49. Remien RH, Stirratt MJ, Dolezal C, et al. Couple-focused support to improve HIV medication adherence: a randomized controlled trial. AIDS 2005;19:807-14.

50. Pradier C, Bentz L, Spire B, et al. Efficacy of an educational and counseling intervention on adherence to highly active antiretroviral therapy: French prospective controlled study. HIV Clin Trials 2003:4:121-31.

51. Hovell MF, Sipan CL, Blumberg EJ, et al. Increasing Latino adolescents' adherence to treatment for latent tuberculosis infection: a controlled trial. Am J Public Health 2003;93:1871-7.

52. Murphy DA, Lu MC, Martin D, et al. Results of a pilot intervention trial to improve antiretroviral adherence among HIV-positive patients. $J$ Assoc Nurses AIDS Care 2002;13:57-69.

53. Peterson AM, Takiya L, Finley R. Meta-analysis of trials of interventions to improve medication adherence. Am J Health Syst Pharm 2003;60:657-65. 


\section{Correction}

Easthall C, Song F, Bhattacharya D. A metaanalysis of cognitive-based behaviour change techniques as interventions to improve medication adherence. BMJ Open 2013;3:e002749. In the process of preparing this material for inclusion in a doctoral thesis, the following minor errors were noted:

- Remien study (tables 1 and 2) - the correct sample size is 215; data were, however, entered correctly.

- Van Es study (table 2) - the correct sample sizes for the intervention and control group are 33 and 34 respectively; data were, however, entered correctly.

- Pradier and Wagner studies - sample size errors were included in the meta-analysis which means slightly different effect sizes were obtained for these studies as summarised in table 1 below which highlights that the erroneous data have made very minor changes to the effect sizes calculated.

- The overall effect size for all studies remains unchanged at $0.34(0.23$ to 0.46$)$.

\begin{tabular}{llll}
\multicolumn{3}{l}{ Summary of changes to sample sizes and effects sizes } \\
\hline & & Pradier et al & Wagner et al \\
\hline Original data included in paper & Total sample size & 202 & 135 \\
& Intervention group & 123 & 154 \\
& Control group & 121 & 76 \\
& Effect size & 0.34 & -0.08 \\
Updated (correct) data & & $(0.02,0.65)$ & $(-0.35,0.20)$ \\
& Total sample size & 202 & 145 \\
& Intervention group & 100 & 97 \\
& Control group & 102 & 48 \\
& Effect size & 0.29 & -0.10 \\
& & $(0.01,0.57)$ & $(-0.45,0.25)$ \\
\hline
\end{tabular}

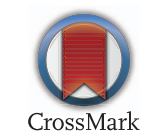

BMJ Open 2014;4:e002749. doi:10.1136/bmjopen-2013-002749corr1 